Published in final edited form as:

Clin Res Hepatol Gastroenterol. 2018 October ; 42(5): 483-493. doi:10.1016/j.clinre.2018.03.003.

\title{
Individuals Affected by Eosinophilic Gastrointestinal Disorders Have Complex Unmet Needs and Frequently Experience Unique Barriers to Care
}

\author{
Girish Hiremath ${ }^{1}$, Ellyn Kodroff ${ }^{2,}$, , Mary J. Strobel ${ }^{3,{ }^{*}}$, Melissa Scott ${ }^{4,}$, Wendy Book ${ }^{3}$, Cathy \\ Reidy $^{4}$, Shay Kyle ${ }^{2}$, Denise Mack ${ }^{3}$, Kathleen Sable ${ }^{3}$, Pablo Abonia $^{5}$, Jonathan Spergel ${ }^{6}$, \\ Sandeep K. Gupta ${ }^{7}$, T. Glenn Furuta ${ }^{8}$, Marc E. Rothenberg ${ }^{5}$, and Evan S. Dellon ${ }^{9}$ \\ ${ }^{1}$ Division of Pediatric Gastroenterology, Hepatology and Nutrition, Monroe Carell Jr Children's \\ Hospital at Vanderbilt, Nashville, TN, USA
}

Corresponding author: Girish S. Hiremath, MD MPH, Assistant Professor of Pediatrics, Division of Pediatric Gastroenterology,
Hepatology \& Nutrition, Monroe Carell Jr. Childrens Hospital at Vanderbilt, DOT Suite 10226, 2200 Childrens Way, Nashville TN
37232, Phone: 615-875-9690, Fax: 615-343-5323, Girish.Hiremath@ Vanderbilt.edu.
These authors all contributed equally to the study.
Publisher's Disclaimer: This is a PDF file of an unedited manuscript that has been accepted for publication. As a service to our Publisher's Disclaimer: This is a PDF file of an unedited manuscript that has been accepted for publication. As a service to our
customers we are providing this early version of the manuscript. The manuscript will undergo copyediting, typesetting, and review of the resulting proof before it is published in its final citable form. Please note that during the production process errors may be discovered which could affect the content, and all legal disclaimers that apply to the journal pertain.

Declarations

Ethics approval and consent to participate:

This study (protocol \# 161510) was approved by the Institutional Review Board at the Vanderbilt University.

Consent for publication

Not applicable

Availability of data and material

All data generated or analyzed during this study are included in this published article [and its supplementary information files].

Competing interests

GSH: The author declares no competing interest

EK: The author declares no competing interest

MJS: The author declares no competing interest

MS: The author declares no competing interest

WB: The author declares no competing interest

CR: The author declares no competing interest

SK: The author declares no competing interest

DM: The author declares no competing interest

KS: The author declares no competing interest

PA: The author declares no competing interest

JS: The author declares no competing interest

SKG: The author declares no competing interest

GTF: No potential conflicts of interest related to this paper. Consultant for Banner and Shire.

MER: No potential conflicts of interest related to this paper. Consultant for NKT Therapeutics, Pulm One, Spoon Guru, Celgene,

Shire, Astra Zeneca, GlaxoSmithKline, Allakos, and Novartis and has an equity interest in the first three listed and Immune

Pharmaceuticals, and royalties from reslizumab (Teva Pharmaceuticals). M.E.R. is an inventor of patents, owned by Cincinnati

Children's.

ESD: No potential conflicts of interest related to this paper. Consultant for Adare, Alivio, Alkalso, Banner, Celgene/Receptos,

Enumeral, GSK, Regeneron, Shire. Research funding from Adaire, Meritage, Miraca, Nutricia, Celegene/Receptos, Regeneron, Shire.

Authors' contributions

GSH, EK, MJS, MS, CR, SK, KS, PA, JS, SKG, MER, ESD: Made substantial contributions to conception and design, or acquisition of data, or analysis and interpretation of data. They were also involved in drafting the manuscript or revising it critically for important intellectual content.

WB, DM, GTF: Made substantial contributions in interpretation of the data and were also involved in drafting the manuscript or revising it critically for important intellectual content. 
${ }^{2}$ Campaign Urging Research for Eosinophilic Diseases, Lincolnshire, IL, USA

${ }^{3}$ American Partnership for Eosinophilic Disorders, Atlanta, GA, United States

${ }^{4}$ Eosinophilic Family Coalition, Cincinnati, OH, USA

${ }^{5}$ Division of Allergy and Immunology, Cincinnati Children's Hospital and Medical Center, Cincinnati, OH, USA

${ }^{6}$ Division of Allergy and Immunology, Department of Pediatrics, Perelman School of Medicine at University of Pennsylvania, Philadelphia, PA, USA

${ }^{7}$ Division of Pediatric Gastroenterology, Hepatology and Nutrition, University of Illinois College of Medicine, Peoria, IL, USA

${ }^{8}$ Digestive Health Institute, Gastrointestinal Eosinophilic Diseases Program, Children's Hospital Colorado, Aurora, Colorado, USA

${ }^{9}$ Center for Esophageal Diseases and Swallowing, Division of Gastroenterology and Hepatology, University of North Carolina School of Medicine, Chapel Hill, NC, USA

\section{Abstract}

Introduction-Eosinophilic gastrointestinal disorders (EGIDs) are a rare but emerging healthcare problem. Patient advocacy groups (PAGs) have an important role in representing the EGID community, and serve as valuable research partners. By leveraging the partnership between medical researchers and PAGs, we examined the unmet needs and barriers to care perceived by individuals affected by EGIDs. Next, we examined if these varied between adult EGID patients and adult caregivers of children with EGID.

Methods-Adult EGID patients and adult caregivers of children ( $<18$ years) with EGIDs participated in this study. PAGs conducted focus groups comprised of individuals affected by EGIDs to identify domains and questions meaningful to the EGID community and this information was used to develop an online REDCap survey. The survey consisted of 58 questions across medical, healthcare, social, and emotional impact domains. It was distributed via the PAGs' web-based platforms. Demographic data, and responses to questions on a six-point Likert scale were collected and analyzed.

Results-Of the 361 responses analyzed, 90 (25\%) were from adult EGID patients and 271 (75\%) were from adult caregivers. Of the applicable responses, in the medical domain only $19 \%$ of participants indicated that repeated endoscopies to monitor response to treatment was convenient. In the healthcare domain, $67 \%$ indicated that lack of insurance coverage for elemental formula was a barrier. In the social domain, only $5 \%$ of respondents reported adequate awareness of EGIDs in schools. In the emotional domain, 64\% had experienced significant stress due to EGID related outof-pocket costs. Multivariate logistic regression revealed that some of these responses varied between adult EGID patients and adult caregivers of children with EGID. The respondents indicated highest priority for improvement in the medical domain compared to other domains.

Conclusions-Individuals affected by EGIDs have a constellation of complex unmet needs and perceived barriers across medical, healthcare, social and emotional domains. Addressing unmet 
needs in the medical domain is relatively more important for the EGID community. Understanding unmet needs and barriers will likely help design improved patient-centered EGID care paradigms.

\section{Keywords}

Rare diseases; Eosinophilic gastrointestinal disorders; Eosinophilic esophagitis; Eosinophilic gastritis; Eosinophilic gastroenteritis; Unmet needs; Survey; Health care barriers; Health services research; Health promotion

\section{INTRODUCTION}

Eosinophilic gastrointestinal disorders (EGIDs) are an emerging group of rare disorders.[1]

Based on the affected site within the gastrointestinal tract, EGIDs are classified as distinct entities, such as eosinophilic esophagitis (EoE), eosinophilic gastritis (EG), eosinophilic colitis (EC); the term eosinophilic gastroenteritis (EGE) is used when multiple segments of the gastrointestinal tract are involved. EoE is the most prevalent and well-studied EGID.[2,3] Despite significant efforts, the process of detecting, monitoring and managing EGIDs remains expensive, complex and cumbersome.[4] It is conceivable that these factors could result in a constellation of unmet needs and barriers to the EGID community and could impact lives of individuals affected by EGIDs, but this has never been formally studied. Patient advocacy groups (PAGs) such as the American Partnership for Eosinophilic Disorders (APFED), Campaign Urging Research for Eosinophilic Diseases (CURED), and the Eosinophilic Family Coalition (EFC), have emerged as key partners in improving the lives of individuals affected by EGIDs.[5] They are deeply involved in raising awareness regarding EGIDs through educating patients, caregivers, health care providers, government, industry representatives, and the general public.[6] PAGs also have the potential to ensure feasibility and success of research protocols by making valuable contributions to study design, and implementation by connecting medical researchers with large numbers of EGID patients and their caregivers worldwide through a variety of avenues including their webbased platforms.

The 3 aims of this cross-sectional study were to: 1) examine the utility of PAG web-based platforms for reaching the dispersed EGID community with an online survey; 2) identify unmet needs and barriers to care perceived by the EGID community in the domains important and relevant to them; and 3) investigate if the perceived unmet need(s) or barrier(s) differed between adult EGID patients and adult caregivers of children with EGID. We hypothesized that the PAG's web-based platforms would be an effective portal to reach the dispersed EGID community, and that the individuals affected by EGIDs would have complex and varied unmet needs spanning across multiple domains and that the perception of unmet needs and barriers would differ between adult patients and adult caregivers of children with EGIDs. 


\section{METHODS}

\section{Subjects}

Adult EGID patients and adult caregivers of children ( $<18$ years of age) diagnosed with EGID, and enrolled in PAG administered web-based platforms (e.g., email list-serve, social media channel) were invited to participate in this study. Adult caregivers were defined as parent(s) or guardian(s) directly involved in providing care to their children diagnosed with EGID. By limiting participation to adult caregivers as opposed to opening participation to the pediatric population, we were able to minimize potential inconsistencies and irregularities associated with varying cognitive abilities among children and young adolescents, and maintain homogeneity in response quality.

\section{Study Design and Setting}

Partnership between medical researchers with expertise in EGIDs and PAGs resulted in development of this study protocol and the survey instrument, as illustrated in Figure 1. This study was approved by the Institutional Review Board at Vanderbilt University (protocol \# 161510).

Focus groups-To identify domains most important and relevant to the EGID community and for deeper exploration of the unmet needs and barriers to care perceived by individuals affected by EGIDs, each PAGs conducted focus groups.[7] Each focus group conducted by APFED consisted of 10 participants ( 3 adult EGID patients, 7 caregivers) recruited from their database. One of the participants was a support group leader for an adult/teen group for EGIDs. Focus groups conducted by CURED consisted of 10 participants ( 3 adult EGID patients, and 7 caregivers), and EFC focus groups consisted of 5 participants ( 3 adult EGID patients, and 2 caregivers). Participants included in the CURED and EFC focus groups were recruited through social media and EGID community group outlets. Each of the three PAGs collected feedback via telephone interview and/or online, consolidated and summarized inputs to best represent the needs addressed in the majority of the comments received. None of the participants contributed to more than one focus group.

Developing the survey instrument-Based on the insights from focus groups, a Research Electronic Data Capture (REDCap) survey was developed.[8] The survey underwent multiple iterations of review and refinement by authors who have expertise in the area of EGIDs and survey design (GH, PA, MR, ED), $[9,10]$ and representatives from PAGs (EK, MJS, DM, CR, KS). The purpose of this iterative process was to ensure that precise and simple language was being used in the questionnaire, and the questions were relevant to study aims. The final REDCap survey was comprised of 5 sections and 58 total questions (see supplementary material Appendix 1). The first section collected demographics and disease characteristics, with no personal identifiers. Subsequent sections gathered information over four domains identified to be most important and relevant to the EGID community: (1) medical challenges, (2) healthcare barriers, (3) social aspects, and (4) emotional impact of living with EGIDs. The responses were collected on a 6-point Likert scale ranked as: (1) Totally Disagree, (2) Disagree, (3) Neutral, (4) Agree, (5) Totally Agree, and (6) Not Applicable. Optional comments were collected via a free-text box. At the end of 
the survey, respondents were asked to rank each of the domains ranging from greatest need for improvement to least or lowest need for improvement (1: greatest need; 2 : some need; 3 : little need; 4: lowest or least need). By allowing respondents to rank each of the domains we were able to simultaneously compare relative proportions for each of the domains. The questionnaire was designed to take less than 15 minutes to complete.

Dissemination of the Survey, Reminders and Data collection-Web-based platforms, including electronic mail and social media channels administered by PAGs were used to recruit participants. Electronic reminders were sent every 2 weeks for a total of 4 reminders, resulting in a data collection period of 8 weeks. The data were collected between October 2016 and December 2016. Survey responses were securely stored on the Vanderbilt University Medical Center server until analysis.

\section{Statistical analysis}

Data were exported from Stata version 14.0 (StataCorp., College Station, TX) for analysis. Descriptive statistics including counts and percentages for categorical variables, and means and standard deviations for continuous variables were used to characterize respondents. We dichotomized the applicable responses from the participants into "Agree" (Strongly agree + Agree) and "Disagree" (Strongly disagree + Disagree + Neutral) categories to investigate the perceived unmet needs and barriers to care. To compare responses between adult EGID patients and adult caregivers of children with EGIDs, unadjusted logistic regression was used to estimate odds ratios (OR) with 95\% confidence intervals (95\% CI) for each question in the survey. Multivariate logistic regression was used to adjust for potential independent predictors such as the type of EGID, duration of disease, country of origin, insurance, and disease activity.

\section{RESULTS}

\section{Demographics}

A total of 414 responses were received. Of these, 53 responses were excluded because 39 were from individuals < 18 years of age and 14 did not specify their age. Of the 361 responses analyzed, 280 (78\%) were complete in all aspects. Nearly 18\% of participants did not respond to at least one of the questions. The rate of incomplete responses was highest in the emotional impact domain at 18\%, and it was lowest for the medical challenges domain at $13 \%$.

Our cohort consisted of 90 (25\%) adult EGID patients, and 271 (75\%) adult caregivers of children with EGIDs. EoE (88\%) was the most common form of EGID reported, and approximately 20\% reported overlapping EGIDs (e.g. EGE). Respondents from 9 countries participated in this study, with the top 3 coming from the United States (US; 90\%), Australia (5\%) and Canada (2\%). Within the US, a majority of the participants resided in the southern region (38\%). Most participants had private insurance (75\%), and only $2 \%$ were uninsured. A notable proportion of participants (76\%) had been diagnosed with EGIDs within the past 5 years. The characteristics of the EGID patient group and the caregiver group were similar in terms of types of EGIDs, country, region within the US, and insurance coverage status 
(Table 1). As expected, significant differences between adult EGID patient group and the caregiver group were noted in age, time since diagnosis of EGID, relationship, and employment status.

\section{Medical challenges}

When asked about the medical challenges, $52 \%$ of participants indicated that they had to change multiple providers prior to being diagnosed with EGID, and 53\% indicated that they had to transfer medical care to a provider knowledgeable in EGID. In all, 78\% indicated that their current EGID providers were knowledgeable about EGIDs, and 64\% reported that their providers had clearly discussed available treatment options for their EGID. Only a small proportion of participants indicated that repeated endoscopies to assess treatment response were convenient (19\%) and affordable (33\%). With regards to currently available treatment options, only $31 \%$ on dietary elimination, $31 \%$ on a combination of swallowed steroids and dietary elimination therapy, $17 \%$ on elemental diet, and $21 \%$ on immunomodulators (e.g., 6 mercaptopurine) indicated that they were easy to adhere and convenient (Table 2a).

The bivariate and multivariate logistic regression revealed that the perception of unmet needs and potential barriers in the medical domain were similar between adult EGID patients and adult caregivers of children with EGIDs (Table 3a).

\section{Healthcare barriers}

In all, $68 \%$ of respondents indicated that their insurance covered the cost of endoscopy to monitor EGIDs, and 58\% indicated that their insurance covered most of the cost of the swallowed steroids required to treat their EGID. However, only one in three respondents $(33 \%)$ indicated that insurance covered the cost of their elemental formula. Approximately $64 \%$ of respondents indicated that they did not have an easy access to dietitians or nutritionists who they felt understood the challenges faced by EGID patients, and 57\% indicated that they had not been evaluated for potential nutritional deficiencies. A majority $(68 \%)$ of respondents indicated that their medical team sought their inputs while making decisions related to their/their child's care (Table $2 b$ ).

There were multiple differences between EGID patients and adult caregivers after bivariate logistic regression, but after multivariate analysis only few persisted. Adult caregivers of children with EGIDs had relatively easier access to dietitians or nutritionists who were familiar with the challenges related to EGIDs compared with adult EGID patients themselves [aOR (95\% CI): $1.22(1.00-1.49)]$, and were more likely to have had their children evaluated by a nutritionist for nutritional deficiencies [1.61 (1.29-2.02)]. The adult caregivers also indicated that their medical providers were relatively more knowledgeable about requirements for special accommodations (e.g., school, disability) [1.44 (1.11 - 1.87)], and were more likely to feel that the medical team encouraged their inputs while making EGID care-related decisions [1.67 (1.27 - 2.20)] (Table 3b).

\section{Social aspects}

The majority (79\%) of respondents indicated that they used social media to connect with peers. Approximately $62 \%$ had experienced food-related discrimination due to dietary 
restrictions to manage their EGID. In all, 83\% reported that they were knowledgeable about preparing allergen-free foods, and $85 \%$ knew where to buy specialty foods that were free from ingredients that they were avoiding. Only a small proportion of respondents indicated that there was adequate awareness about EGIDs in schools (5\%) and work places (6\%), and that teachers or employers were empathetic to their needs (Table 2c).

Multivariate logistic regression revealed that compared to the adult EGID patients, the caregivers of children with EGIDs knew where to buy specialty foods [1.55 $(1.13-2.12)]$, and had supportive immediate $[1.30(1.01-1.67)]$ and extended family members [1.44 (1.11 - 1.86)] (Table 3c).

\section{Emotional impact}

In all, 70\% indicated that they had support through their family and friends. Over half of the respondents (51\%) indicated that they felt the need to seek professional help to manage emotional stress related to EGIDs, and $41 \%$ had actually sought professional help. Only $17 \%$ indicated that they had a local EGID support group in which they could participate. The majority of the participants indicated that their EGID had placed an emotional burden on family members (74\%) and caregivers (75\%), and 64\% indicated that the out-of-pocket costs related to EGID had placed significant stress on them (Table 2d).

Multivariate logistic regression revealed that caregivers of children with EGIDs had actually sought help from a professional therapist to cope with emotional stress related to EGID [1.36 (1.07 - 1.73)], and that the EGID had placed emotional burden on family members [1.62 (1.46-2.71)] and on caregivers [1.99 $(1.46-2.13)]$ (Table 3d).

\section{Ranking the domains for improvement}

As illustrated in the radar plot (Figure 2), 33\% indicated that there was greatest need for improvement in the medical domain, followed by the healthcare challenges (32\%) and emotional impact of EGIDs domain (20\%). The least amount of improvement (33\%) was needed in the social challenges domain. The ranking of domains was identical between adult EGID patients and adult caregivers of children with EGIDs.

\section{DISCUSSION}

This is the first study to investigate unmet needs and barriers perceived by those affected by the rare group of diseases, EGIDs, in the domains meaningful to them. Our results indicate that individuals affected by EGIDs have complex unmet needs across medical, healthcare, social, and emotional domains, and the perception of some of the unmet needs vary between adult EGID patients and adult caregivers of children with EGIDs.

By leveraging partnership between medical researchers and PAGs, this study achieved participation from a large and diverse group of adult EGID patients and adult caregivers of children with EGIDs. These observations suggest that the internet-based research can be effectively used to complement information generated through traditional healthcare and public health systems, and that the individuals affected by rare diseases including EGIDs can 
be highly motivated internet users tapping into online supportive networks, and are receptive to novel web-based research.[11,12]

Unfortunately, more than half of the EGID patients often transition through multiple providers to find a physician knowledgeable in EGIDs and/or experience delays in diagnosis. This is likely related to relatively few centers offering comprehensive and integrated care to EGID patients. While there are multiple therapeutic options to manage EGIDs, $[13,14]$ the decision-making process leading up to the preferred treatment pathway can be complicated. Furthermore, the therapy in itself can be burdensome and expensive, especially if patients lack adequate insurance coverage.[15,16] For many EGID patients, the dietary allergen elimination is an effective therapeutic option, and dietitians play an important role in assuring that EGID patients and their caregivers are informed about the suspected ingredient(s) to avoid and assessing for adequate nutritional status of individuals affected by EGIDs.[17] Our results indicate that limited access to dietitians knowledgeable about EGIDs and not being evaluated for nutritional deficiencies are important unmet needs among those who participated in this study. Our data also highlights the perceived lack of awareness about EGIDs in schools, workplaces, and among family members, as well as the emotional burden EGIDs place on the patients and their caregivers.

This study has limitations. We targeted an online EGID community by leveraging social media platforms administered by three prominent PAGs that serve the EGID population. While this recruitment approach offered us a distinct advantage of reaching out to diverse and dispersed group of individuals affected by EGIDs, it is possible that the same approach also served as a source for introducing potential bias that could limit generalizability of our results. Engaging individuals participating in support groups or advocacy groups could potentially represent those who have ready access to internet, those likely struggling to deal with their condition, and perhaps those more inclined to seek out forums and web-based support. Their responses may not reflect the experiences of a broader EGID community. The use of internet and web-enabled platforms provide an unprecedented opportunity to conduct clinical trials and natural history studies, and educate stakeholders.[18] Moreover, the anonymity of participating in online surveys and self-reporting can be an asset for eliciting information that patients may not feel comfortable discussing with their healthcare providers. However, these methods do not allow us to confirm medical and demographic information provided by the respondents, and do not allow us to reliably calculate a survey response rate. Our study has several strengths. We adapted a community-based participatory research framework wherein the PAGs were actively involved in all aspects of the study.[19] Use of social media outlets to disseminate the survey questionnaire, robust methodology, a large sample size, and rigorous analytical approach further strengthened our study.

In conclusion, EGIDs are receiving more attention due to increased recognition in the healthcare community, improved understanding of the underlying pathophysiology, advances to bring better therapies to market, and greater public awareness. However, individuals affected by EGIDs continue to face a variety of challenges including lack of specialized care, delays in diagnosis, financial burden, negative social consequences, psychosocial ramifications and emotional burden. Our study illustrates a spectrum of complex unmet needs and barriers across multiple domains relevant to the EGID community. Understanding 
the constellation of unmet needs and barriers perceived by the EGID community will likely serve as an important step towards further identification and prioritization of their needs, inform evidence-based policy interventions to bridge gaps, and inspire future patientcentered research to complement ongoing biomedical research.

\section{Supplementary Material}

Refer to Web version on PubMed Central for supplementary material.

\section{Acknowledgments}

Funding and Acknowledgement

All authors are affiliated with the Consortium of Eosinophilic Gastrointestinal Disorders Researchers (CEGIR). CEGIR (U54 AI117804) is part of the Rare Disease Clinical Research Network (RDCRN), an initiative of the Office of Rare Diseases Research (ORDR), NCATS, and is funded through collaboration between NIAID, NIDDK, and NCATS. CEGIR is also supported by patient advocacy groups including APFED, CURED and EFC. G.H is also supported by a grant from the American Partnership for Eosinophilic Disorders (APFED).

\section{References}

1. Rothenberg ME. Eosinophilic gastrointestinal disorders (EGID)*. Journal of Allergy and Clinical Immunology. 2004; 113:11-28. [PubMed: 14713902]

2. Jensen ET, Martin CF, Kappelman MD, Dellon ES. Prevalence of Eosinophilic Gastritis, Gastroenteritis, and Colitis: Estimates From a National Administrative Database. Journal of Pediatric Gastroenterology and Nutrition. 2016; 62:36-42. [PubMed: 25988554]

3. Dellon ES, Jensen ET, Martin CF, Shaheen NJ, Kappelman MD. Prevalence of eosinophilic esophagitis in the United States. Clin. Gastroenterol. Hepatol. 2014; 12:589-96. e1. [PubMed: 24035773]

4. Fahey LM, Liacouras CA. Eosinophilic Gastrointestinal Disorders. Pediatr. Clin. North Am. 2017; 64:475-85. [PubMed: 28502433]

5. Cheng K, Gupta SK, Kantor S, Kuhl J, Aceves S, Bonis P. , et al. Translational Science of Rare Diseases. IOS Press; Creating a multicenter rare disease consortium - the consortium of eosinophilic gastrointestinal disease researchers (CEGIR); 1-15. Preprint

6. Terry SF. Disease advocacy organizations catalyze translational research. Front Genet. Frontiers. 2013:4.

7. Forsythe LP, Szydlowski V, Murad MH, Ip S, Wang Z, Elraiyah TA, et al. A Systematic Review of Approaches for Engaging Patients for Research on Rare Diseases. J GEN INTERN MED. 2014; 29:788-800. [PubMed: 24113807]

8. Harris PA, Taylor R, Thielke R, Payne J, Gonzalez N, Conde JG. Research electronic data capture (REDCap)_A metadata-driven methodology and workflow process for providing translational research informatics support. Journal of Biomedical Informatics. 2009; 42:377-81. [PubMed: 18929686]

9. Dellon ES, Collins MH, Bonis PA, Leung J, Capocelli KE, Dohil R, et al. Substantial Variability in Biopsy Practice Patterns Among Gastroenterologists for Suspected Eosinophilic Gastrointestinal Disorders. Clin. Gastroenterol. Hepatol. 2016; 14:1842-4. [PubMed: 27112108]

10. Franciosi JP, Hommel KA, DeBrosse CW, Greenberg AB, Greenler AJ, Abonia JP. , et al. Child: Care, Health and Development. Vol. 38. Blackwell Publishing Ltd; 2012. Quality of life in paediatric eosinophilic oesophagitis: what is important to patients?; 477-83.

11. Baker L, Wagner TH, Singer S, Bundorf MK. Use of the Internet and E-mail for Health Care Information: Results From a National Survey. JAMA. American Medical Association. 2003; 289:2400-6.

12. Dwyer AA, Quinton R, Morin D, Pitteloud N. Identifying the unmet health needs of patients with congenital hypogonadotropic hypogonadism using a web-based needs assessment: implications for 
online interventions and peer-to-peer support. Orphanet J Rare Dis. 2014; 9:83. [PubMed: 24915927]

13. Aceves S, Hirano I, Furuta GT, Collins MH. Semin Immunopathol. Vol. 34. Springer-Verlag; 2012. Eosinophilic gastrointestinal diseases_clinically diverse and histopathologically confounding; 715-31.

14. Shah NA, Albert DM, Hall NM, Moawad FJ. Clin Exp Gastroenterol. Vol. 9. Dove Press; 2016. Managing eosinophilic esophagitis: challenges and solutions; 281-90.

15. Gonzalez-Cervera J, Lucendo AJ. Eosinophilic Esophagitis: An Evidence-Based Approach to Therapy. J Investig Allergol Clin Immunol. 2016; 26 8-18-quiz2pfollowing 18.

16. Cotton CC, Erim D, Eluri S, Palmer SH, Green DJ, Wolf WA, et al. Cost Utility Analysis of Topical Steroids Compared With Dietary Elimination for Treatment of Eosinophilic Esophagitis. Clinical Gastroenterology and Hepatology. 2016

17. Venter C, Fleischer DM. Annals of Allergy, Asthma \& Immunology. Vol. 117. Elsevier; 2016. Diets for diagnosis and management of food allergy; 468-71.

18. Merkel PA, Manion M, Gopal-Srivastava R, Groft S, Jinnah HA, Robertson D, et al. The partnership of patient advocacy groups and clinical investigators in the rare diseases clinical research network. Orphanet J Rare Dis. BioMed Central. 2016; 11:66.

19. Esperat MCR, Feng D, Owen DC, Green AE. Transformation for health: A framework for health disparities research. Nursing Outlook. 2005; 53:113-20. [PubMed: 15988447] 


\section{Highlights}

Eosinophilic gastrointestinal disorders (EGID) are group of rare but pose significant burden worldwide

Patient advocacy groups have an important role in representing the EGID community

This is the first study to use online portal to study unmet needs and barriers experienced by individuals affected by EGIDs

Our results reveal that individuals affected by EGIDs have a constellation of complex unmet needs and perceived barriers across medical, healthcare, social and emotional domains

Understanding unmet needs and barriers will likely guide us to design EGID patient-centered approaches to improve their care and clinical outcomes 


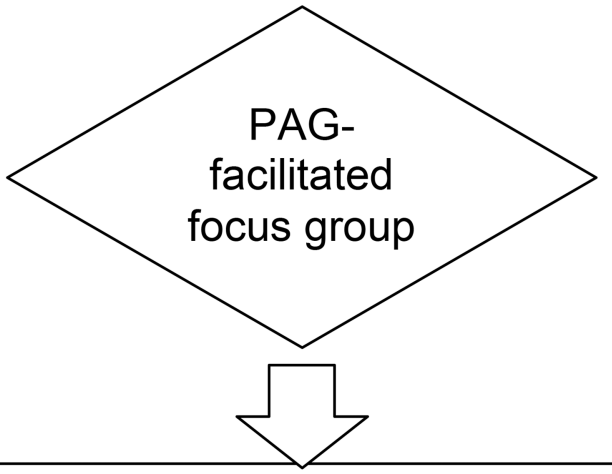

1. Identifying domains relevant and important to the EGID community

2. Within each domain, recognize questions which may be relevant and important to EGID community
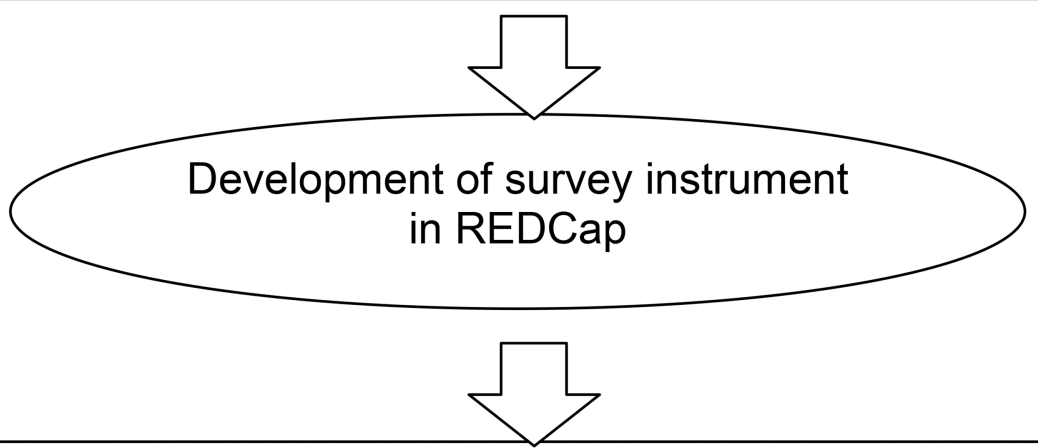

Finalization of survey instrument based on collaborative PAG and researcher input

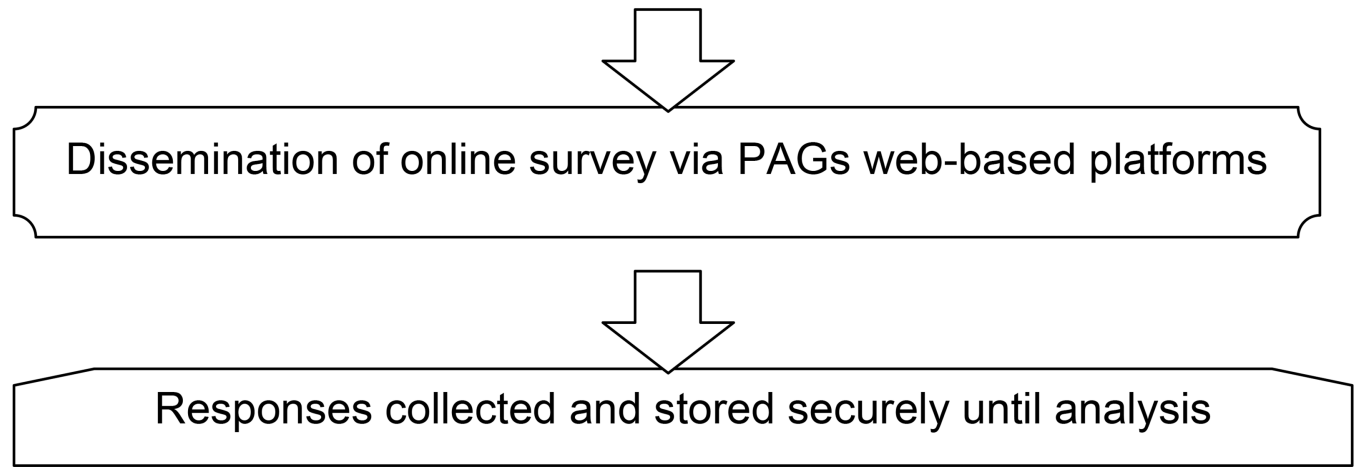

Figure 1.

Schematic representation of the study protocol 


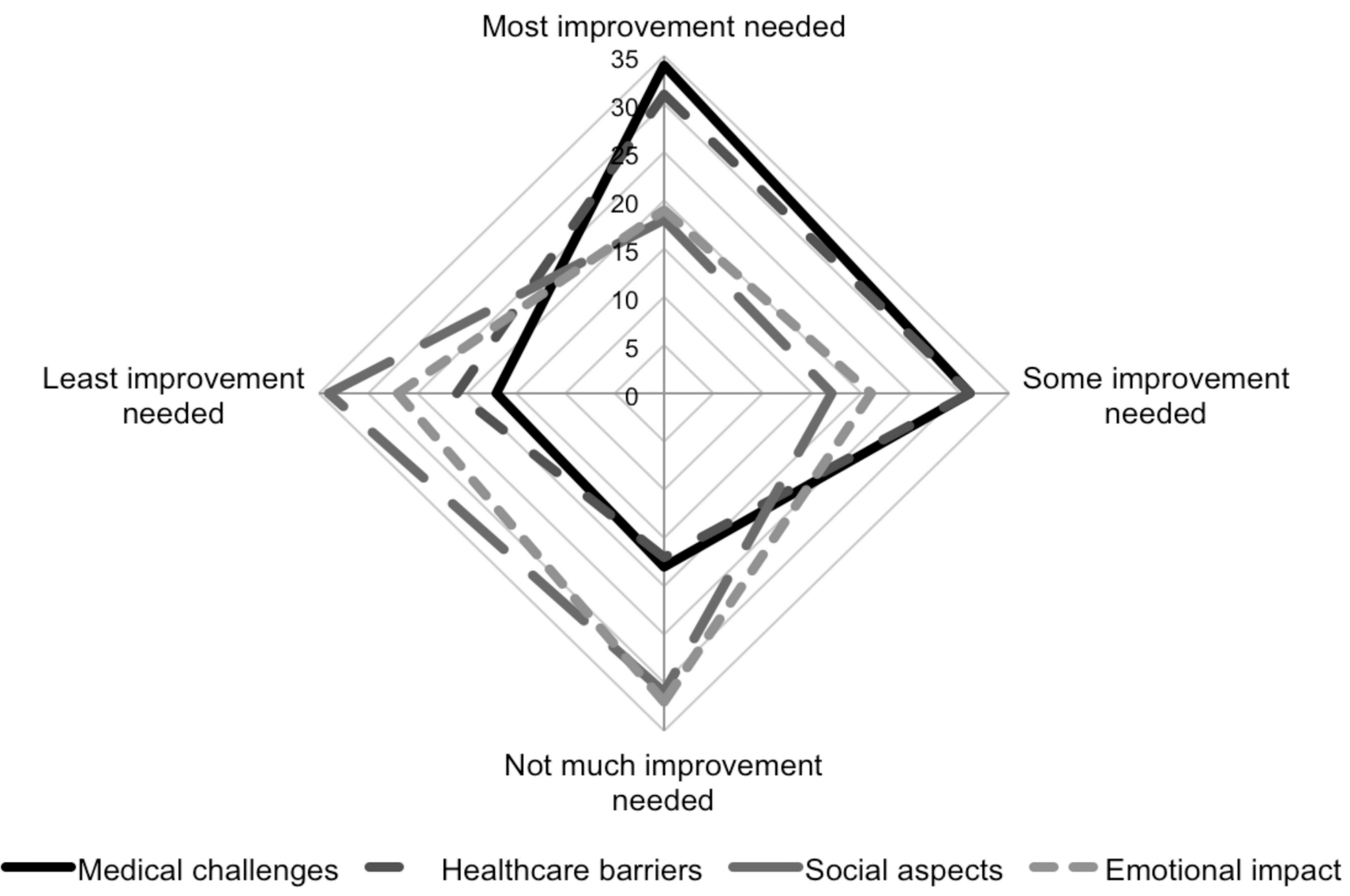

Figure 2.

Radar plot illustrating priority for improvement assigned to each domain by the participants 


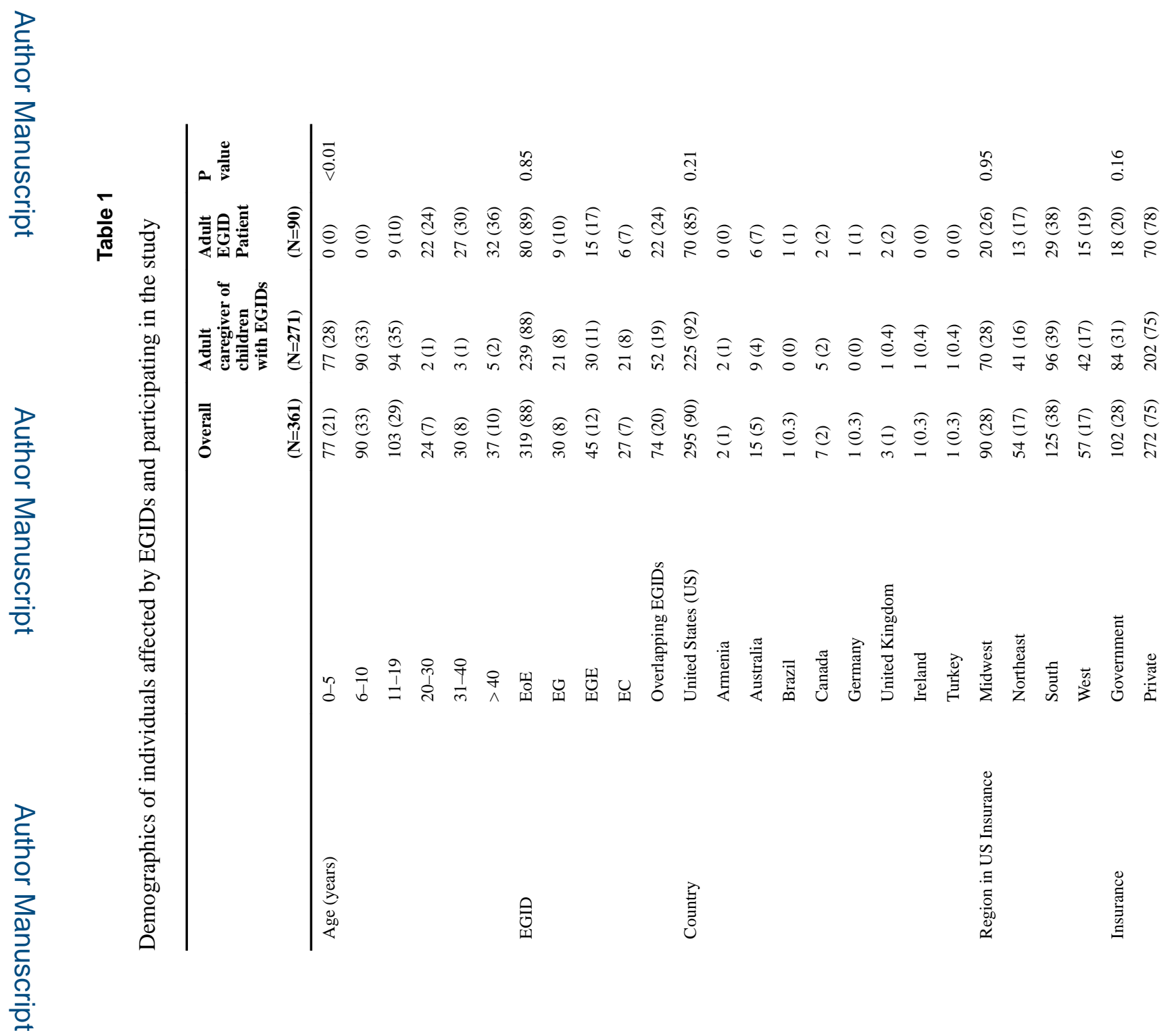

Clin Res Hepatol Gastroenterol. Author manuscript; available in PMC 2019 October 01. 

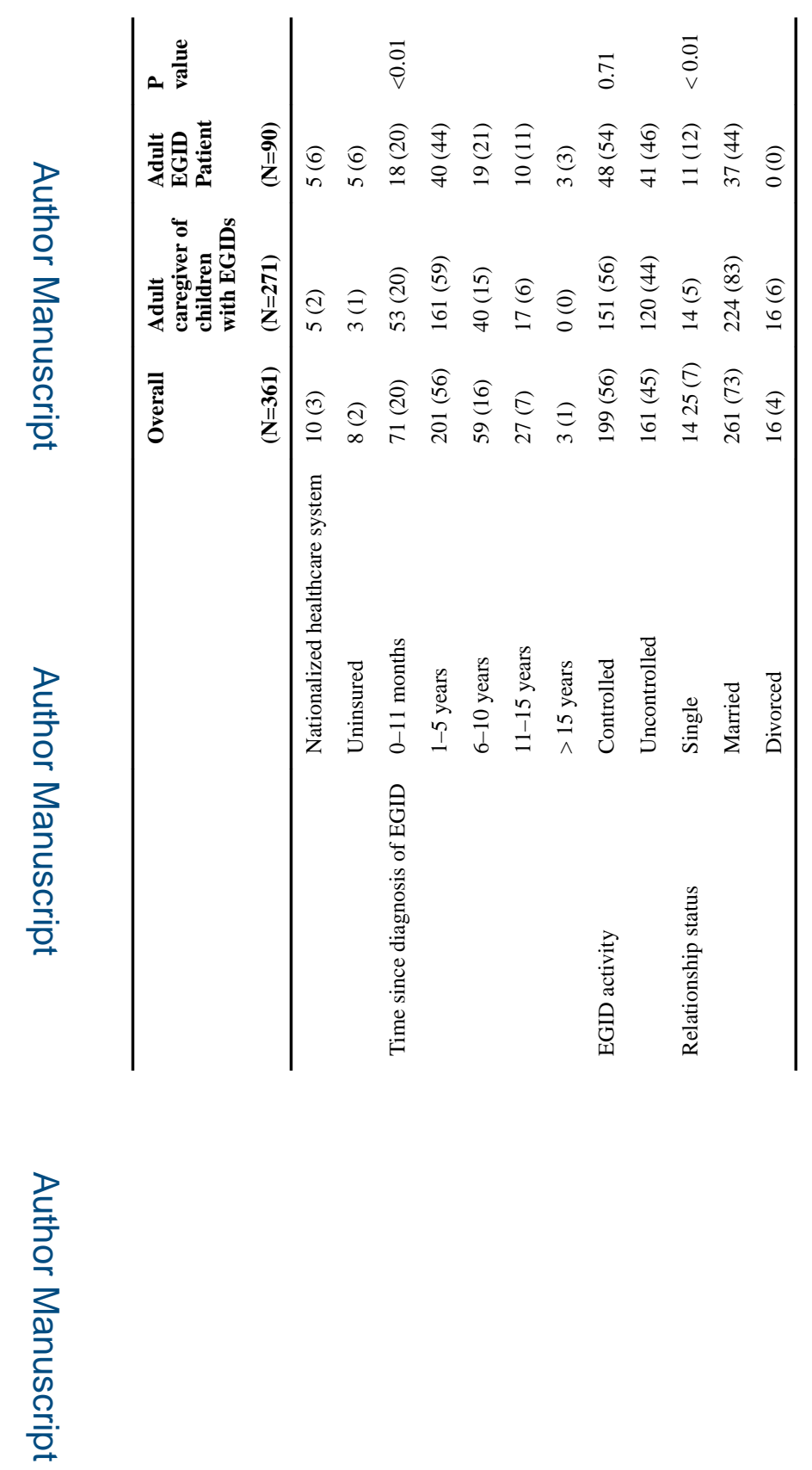

로을

Clin Res Hepatol Gastroenterol. Author manuscript; available in PMC 2019 October 01. 
Table 2

Challenges and barriers perceived by the participating adult EGID patients and adult caregivers of children with EGID

\begin{tabular}{|c|c|c|c|}
\hline \multirow[t]{2}{*}{ Question } & \multicolumn{3}{|c|}{ Applicable responses } \\
\hline & $\begin{array}{l}\text { Out of a total } \\
\text { of } 361 \\
\text { responses } \\
N(\%)\end{array}$ & $\begin{array}{l}\text { Agree } \\
\text { (Strongly agree } \\
+ \text { Agree) } \\
\mathrm{N}(\%)\end{array}$ & $\begin{array}{l}\text { Disagree } \\
\text { (Strongly } \\
\text { Disagree + } \\
\text { Disagree + } \\
\text { Neutral) } \\
\text { N }(\%)\end{array}$ \\
\hline At the time of diagnosis, my medical providers were knowledgeable about EGIDs & $322(89)$ & $162(50)$ & $160(50)$ \\
\hline I was referred to a GI doctor fairly quickly for a diagnosis of EGID & $313(87)$ & $116(37)$ & $197(61)$ \\
\hline I had to change multiple providers prior to being diagnosed with EGID & $308(85)$ & $161(52)$ & $147(48)$ \\
\hline I had to transfer medical care in order to find a provider knowledgeable in EGID & $302(84)$ & $160(53)$ & $142(47)$ \\
\hline My current EGID provider is knowledgeable about EGIDs & $315(87)$ & $246(78)$ & $69(22)$ \\
\hline My team of providers clearly discussed all available treatment options & $316(88)$ & $203(64)$ & $113(36)$ \\
\hline My team of providers gave me adequate information about the sources of allergens & $307(85)$ & $159(52)$ & $148(48)$ \\
\hline Getting repeated endoscopies to see if I am responding to treatment is convenient & $307(85)$ & $58(19)$ & $249(81)$ \\
\hline I can afford repeated endoscopies to see if I am responding to treatment & $301(83)$ & $100(33)$ & $201(67)$ \\
\hline $\begin{array}{l}\text { I am currently on dietary elimination treatment only (such as 4- or 6-food } \\
\text { elimination, or allergy test based elimination) and I find this treatment to be easy to } \\
\text { adhere to and convenient }\end{array}$ & $241(67)$ & $75(31)$ & $166(69)$ \\
\hline $\begin{array}{l}\text { I am currently on swallowed steroids (such as Flovent or Budesonide) and I find } \\
\text { this treatment to be easy to adhere to and convenient }\end{array}$ & $194(54)$ & $93(48)$ & $101(52)$ \\
\hline $\begin{array}{l}\text { I am currently on a combination of swallowed steroids and dietary elimination } \\
\text { therapy and I find this treatment to be easy to adhere to and convenient }\end{array}$ & $193(54)$ & $61(31)$ & $132(69)$ \\
\hline $\begin{array}{l}\text { I am currently on a elemental diet and I find this treatment to be easy to adhere to } \\
\text { and convenient }\end{array}$ & $169(47)$ & $28(17)$ & $141(83)$ \\
\hline I prefer to take steroids over food allergen elimination & $254(70)$ & $78(31)$ & $176(69)$ \\
\hline $\begin{array}{l}\text { I am currently on other medical treatment such as immune modulators (e.g.; 6- } \\
\text { mercaptopurine) and not on local steroids or dietary elimination for treatment of my } \\
\text { EGID and I find this treatment to be easy to adhere to and convenient }\end{array}$ & $151(42)$ & $31(21)$ & $120(79)$ \\
\hline \multicolumn{4}{|l|}{$\underline{\text { Healthcare barriers }}$} \\
\hline \multirow[t]{2}{*}{ Question } & \multicolumn{3}{|c|}{ Applicable responses } \\
\hline & $\begin{array}{l}\text { Out of a total } \\
\text { of } 361 \\
\text { responses } \\
\mathrm{N}(\%)\end{array}$ & $\begin{array}{l}\text { Agree } \\
\text { (Strongly agree } \\
+ \text { Agree) } \\
\mathrm{N}(\%)\end{array}$ & $\begin{array}{l}\text { Disagree } \\
\text { (Strongly Disagree } \\
+ \\
\text { Disagree }+ \\
\text { Neutral) } \\
\mathrm{N}(\%)\end{array}$ \\
\hline $\begin{array}{l}\text { My insurance covers the cost of my endoscopies that are needed to monitor my } \\
\text { EGID }\end{array}$ & $292(81)$ & $199(68)$ & $93(32)$ \\
\hline $\begin{array}{l}\text { My insurance covers most of the cost of the swallowed steroids required to treat my } \\
\text { EGID }\end{array}$ & $221(61)$ & $127(58)$ & $94(42)$ \\
\hline $\begin{array}{l}\text { My insurance covers most of the cost of elemental formula required to treat my } \\
\text { EGID }\end{array}$ & $167(46)$ & $55(33)$ & $112(67)$ \\
\hline $\begin{array}{l}\text { I have easy access to dietitians or nutritionists who understand the challenges of my } \\
\text { EGID }\end{array}$ & $290(80)$ & $104(36)$ & $186(64)$ \\
\hline I have been evaluated by a nutritionist for nutritional deficiencies & $284(77)$ & $123(43)$ & $161(57)$ \\
\hline
\end{tabular}

Clin Res Hepatol Gastroenterol. Author manuscript; available in PMC 2019 October 01. 
Medical domain

Question

Applicable response

\begin{tabular}{|c|c|c|c|}
\hline & $\begin{array}{l}\text { Out of a total } \\
\text { of } 361 \\
\text { responses } \\
N(\%)\end{array}$ & $\begin{array}{l}\text { Agree } \\
\text { (Strongly agree } \\
+ \text { Agree) } \\
\mathrm{N}(\%)\end{array}$ & $\begin{array}{l}\text { Disagree } \\
\text { (Strongly } \\
\text { Disagree + } \\
\text { Disagree + } \\
\text { Neutral) } \\
\text { N }(\%)\end{array}$ \\
\hline $\begin{array}{l}\text { My medical providers are knowledgeable about requirements for special } \\
\text { accommodations (e.g., school, disability) }\end{array}$ & $262(73)$ & $108(41)$ & $154(59)$ \\
\hline $\begin{array}{l}\text { My medical team encourages my input when making decisions about my/my } \\
\text { child's care }\end{array}$ & $289(80)$ & $198(68)$ & $91(32)$ \\
\hline \multicolumn{4}{|l|}{ Social aspects } \\
\hline \multirow[t]{2}{*}{ Question } & \multicolumn{3}{|c|}{ Applicable responses } \\
\hline & $\begin{array}{l}\text { Out of a total } \\
\text { of } 361 \\
\text { responses } \\
\mathrm{N}(\%)\end{array}$ & $\begin{array}{l}\text { Agree } \\
\text { (Strongly agree } \\
+ \text { Agree) } \\
\mathrm{N}(\%)\end{array}$ & $\begin{array}{l}\text { Disagree } \\
\text { (Strongly Disagree } \\
+ \\
\text { Disagree }+ \\
\text { Neutral) } \\
\mathrm{N}(\%)\end{array}$ \\
\hline I often use social media to connect with others who live with EGIDs & $296(82)$ & $232(79)$ & $64(21)$ \\
\hline $\begin{array}{l}\text { I have experienced food-related discrimination due to my dietary restrictions for } \\
\text { EGID }\end{array}$ & $286(79)$ & $179(62)$ & $107(38)$ \\
\hline I am knowledgeable about preparing allergy-free foods & $293(81)$ & $244(83)$ & $49(17)$ \\
\hline I know where to buy specialty foods & $291(81)$ & $248(85)$ & $43(15)$ \\
\hline There is enough awareness in schools about EGIDs and the teachers are empathetic & $274(76)$ & $12(5)$ & $262(95)$ \\
\hline $\begin{array}{l}\text { There is enough awareness in the work-place about EGIDs and the employers are } \\
\text { empathetic }\end{array}$ & $238(66)$ & $14(6)$ & $224(94)$ \\
\hline $\begin{array}{l}\text { My immediate family members (or the people living in my home) understand EGID } \\
\text { and are supportive }\end{array}$ & $293(81)$ & $213(73)$ & $80(27)$ \\
\hline My extended family members understand EGID and are supportive & $293(81)$ & $111(38)$ & $182(62)$ \\
\hline \multicolumn{4}{|l|}{ Emotional impact } \\
\hline \multirow[t]{2}{*}{ Question } & \multicolumn{3}{|c|}{ Applicable responses } \\
\hline & $\begin{array}{l}\text { Out of a total } \\
\text { of } 361 \\
\text { responses } \\
\mathrm{N}(\%)\end{array}$ & $\begin{array}{l}\text { Agree } \\
\text { (Strongly agree } \\
+ \text { Agree) } \\
\mathrm{N}(\%)\end{array}$ & $\begin{array}{l}\text { Disagree } \\
\text { (Strongly Disagree } \\
+ \\
\text { Disagree }+ \\
\text { Neutral) } \\
\mathrm{N}(\%)\end{array}$ \\
\hline $\begin{array}{l}\text { I have emotional support through my family and friends to help with me manage } \\
\text { my EGID }\end{array}$ & $293(81)$ & $205(70)$ & $88(30)$ \\
\hline $\begin{array}{l}\text { I have felt the need to seek help from a professional therapist to manage emotional } \\
\text { stress related to my EGID }\end{array}$ & $267(74)$ & $138(51)$ & $129(49)$ \\
\hline $\begin{array}{l}\text { I have actually sought help from professional therapist to cope with my emotional } \\
\text { stress related to EGID }\end{array}$ & $246(68)$ & $100(41)$ & $146(59)$ \\
\hline I have ongoing emotional toll related to my EGID & $274(76)$ & $174(64)$ & $100(36)$ \\
\hline I have a local EGID support group that I participate in & $249(69)$ & $43(17)$ & $206(83)$ \\
\hline My EGID has placed an emotional burden on my family members & $287(80)$ & $212(74)$ & $75(26)$ \\
\hline My EGID has placed an emotional burden on my caregivers & $257(71)$ & $193(75)$ & $64(25)$ \\
\hline The out of pocket cost related to EGID has placed significant stress on me & $278(77)$ & $177(64)$ & $101(36)$ \\
\hline
\end{tabular}

Clin Res Hepatol Gastroenterol. Author manuscript; available in PMC 2019 October 01. 


\section{Table 3}

Predictors for positive perception (an agreement) to the survey questions by adult caregivers of children with EGIDs compared to adult EGID patients based on logistic regression analysis.

\section{Medical domain}

\begin{tabular}{|c|c|c|}
\hline Questions & OR $(95 \% \mathrm{CI})$ & Adjusted OR* $(95 \% \mathrm{CI})$ \\
\hline At the time of diagnosis, my medical providers were knowledgeable about EGIDs & $1.13(0.95-1.35)$ & $1.05(0.86-1.28)$ \\
\hline I was referred to a GI doctor fairly quickly for a diagnosis of EGID & $0.90(0.95-1.34)$ & $0.88(0.73-1.07)$ \\
\hline I had to change multiple providers prior to being diagnosed with EGID & $1.12(0.85-2.43)$ & $1.11(0.91-1.35)$ \\
\hline I had to transfer medical care in order to find a provider knowledgeable in EGID & $1.02(0.86-1.21)$ & $1.04(0.86-1.26)$ \\
\hline My current EGID provider is knowledgeable about EGIDs & $1.21(0.96-1.53)$ & $1.20(0.92-1.57)$ \\
\hline My team of providers clearly discussed all available treatment options & $1.32(1.08-1.61)$ & $1.22(0.96-1.55)$ \\
\hline My team of providers gave me adequate information about the sources of allergens & $1.38(1.13-1.68)$ & $1.24(0.99-1.56)$ \\
\hline Getting repeated endoscopies to see if I am responding to treatment is convenient & $0.89(0.72-1.10)$ & $0.88(0.69-1.12)$ \\
\hline I can afford repeated endoscopies to see if I am responding to treatment & $1.00(0.83-1.21)$ & $0.91(0.73-1.14)$ \\
\hline $\begin{array}{l}\text { I am currently on dietary elimination treatment only (such as 4- or 6-food elimination, or } \\
\text { allergy test based elimination) and I find this treatment to be easy to adhere to and convenient }\end{array}$ & $1.07(0.87-1.31)$ & $1.10(0.87-1.40)$ \\
\hline $\begin{array}{l}\text { I am currently on swallowed steroids (such as Flovent or Budesonide) and I find this } \\
\text { treatment to be easy to adhere to and convenient }\end{array}$ & $1.02(0.83-1.25)$ & $0.99(0.76-1.28)$ \\
\hline $\begin{array}{l}\text { I am currently on a combination of swallowed steroids and dietary elimination therapy and I } \\
\text { find this treatment to be easy to adhere to and convenient }\end{array}$ & $1.01(0.80-1.26)$ & $0.97(0.84-1.12)$ \\
\hline $\begin{array}{l}\text { I am currently on a elemental diet and I find this treatment to be easy to adhere to and } \\
\text { convenient }\end{array}$ & $1.03(0.77-1.40)$ & $0.98(0.66-1.45)$ \\
\hline I prefer to take steroids over food allergen elimination & $1.06(0.87-1.29)$ & $0.98(0.79-1.22)$ \\
\hline $\begin{array}{l}\text { I am currently on other medical treatment such as immune modulators (e.g.; 6- } \\
\text { mercaptopurine) and not on local steroids or dietary elimination for treatment of my EGID } \\
\text { and I find this treatment to be easy to adhere to and convenient }\end{array}$ & $0.90(0.70-1.16)$ & $0.85(0.61-1.19)$ \\
\hline
\end{tabular}

Healthcare barriers

Questions

OR $(95 \% \mathrm{CI})$

Adjusted OR * $(95 \% \mathrm{CI})$

My insurance covers the cost of my endoscopies that are needed to monitor my EGID

$\begin{array}{ll}1.09(0.88-1.33) & 0.92(0.72-1.19) \\ 1.29(1.02-1.62) & 1.19(0.89-1.58) \\ 1.41(1.04-1.90) & 1.21(0.82-1.78) \\ 1.37(1.12-1.67) & 1.22(1.00-1.49) \\ 1.54(1.26-1.88) & 1.61(1.29-2.02) \\ 1.63(1.29-2.05) & 1.44(1.11-1.87) \\ 1.58(1.26-1.99) & 1.67(1.27-2.20)\end{array}$

My insurance covers most of the cost of the swallowed steroids required to treat my EGID

My insurance covers most of the cost of elemental formula required to treat my EGID

I have easy access to dietitians or nutritionists who understand the challenges of my EGID

I have been evaluated by a nutritionist for nutritional deficiencies

My medical providers are knowledgeable about requirements for special accommodations (e.g., school, disability)

My medical team encourages my input when making decisions about my/my child's care

$1.58(1.26-1.99) \quad 1.67(1.27-2.20)$

\section{Social aspects}

\begin{tabular}{|c|c|c|}
\hline Questions & OR $(95 \% \mathrm{CI})$ & Adjusted OR ${ }^{*}(95 \% \mathrm{CI})$ \\
\hline I often use social media to connect with others who live with EGIDs & $1.31(1.05-1.64)$ & $1.31(1.02-1.70)$ \\
\hline I have experienced food-related discrimination due to my dietary restrictions for EGID & $0.98(0.79-1.22)$ & $1.09(0.85-1.40)$ \\
\hline I am knowledgeable about preparing allergy-free foods & $1.31(1.01-1.70)$ & $1.21(0.87-1.68)$ \\
\hline I know where to buy specialty foods & $1.48(1.14-1.92)$ & $1.55(1.13-2.12)$ \\
\hline
\end{tabular}

I know where to buy specialty foods

$1.48(1.14-1.92) \quad 1.55(1.13-2.12)$

Clin Res Hepatol Gastroenterol. Author manuscript; available in PMC 2019 October 01. 
$\underline{\text { Medical domain }}$

\section{Questions}

There is enough awareness in schools about EGIDs and the teachers are empathetic

There is enough awareness in the work-place about EGIDs and the employers are empathetic

My immediate family members (or the people living in my home) understand EGID and are supportive

My extended family members understand EGID and are supportive

\section{Emotional impact:}

Questions

I have emotional support through my family and friends to help with me manage my EGID

I have felt the need to seek help from a professional therapist to manage emotional stress related to my EGID

I have actually sought help from professional therapist to cope with my emotional stress related to EGID

I have ongoing emotional toll related to my EGID

I have a local EGID support group that I participate in

My EGID has placed an emotional burden on my family members

My EGID has placed an emotional burden on my caregivers

The out of pocket costs related to EGID has placed significant stress on my family

*

Adjusted for type of EGID, duration of disease, country of origin, insurance, and disease activity

\section{OR $(95 \% \mathrm{CI})$}

Adjusted OR* (95\% CI)

$0.90(0.65-1.24) \quad 0.97(0.65-1.45)$

$0.88(0.65-1.18) \quad 0.78(0.55-1.10)$

$1.38(1.11-1.71) \quad 1.30(1.01-1.67)$

$1.51(1.20-1.90) \quad 1.44(1.11-1.86)$

OR $(95 \% \mathrm{CI})$

Adjusted OR * $(95 \%$ CI)

$1.35(1.07-1.71) \quad 1.29(0.98-1.70)$

$1.26(1.07-1.48) \quad 1.24(0.97-1.58)$

$1.21(0.98-1.48) \quad 1.36(1.07-1.73)$

$1.18(0.94-1.48) \quad 1.26(0.96-1.64)$

$1.30(1.00-1.69) \quad 1.33(0.98-1.79)$

$1.47(1.17-1.86) \quad 1.62(1.24-2.13)$

$1.86(1.45-2.38) \quad 1.99(1.46-2.71)$

$1.16(0.93-1.44) \quad 1.26(0.98-1.64)$ 